\title{
Estrategias de enseñanza y tutoria docente en estudiantes V ciclo del Instituto de Educación Superior Tecnológico “Huando” - Huaral, 2018
}

\section{Teaching strategies and teaching tutoring in students $\mathbf{V}$ cicle of the Huando - Huaral Public Technology higher Technical Institute - Lima. 2018}

\author{
Marleny Maribel Welson Valencia de Calderón ${ }^{1}$
}

\section{RESUMEN}

Objetivo: Demostrar la relación existente entre las Estrategias de Enseñanza y la Tutoría Docente en estudiantes IX ciclo del Instituto Superior Tecnológico Público Huando - Huaral - Lima, 2018. Material y Métodos: Se planteó el enfoque cuantitativo de diseño correlacional, cuya información fue recolectaba a través de dos cuestionarios aplicados a la muestra integrado por 57 estudiantes. Asimismo, se garantizó su confiabilidad mediante la prueba de Alfa de Cronbach, además de aplicar el paquete computacional SPSS versión 21, con el empleo de la fórmula de Rho de Spearman con un margen de error al 5\%. Resultados: La hipótesis general representado por las variables de estudio encontró una correlación buena de Rho $=.734$, ya que la mayoría consideró que las estrategias de enseñanza son aplicadas casi siempre, lo cual incide en la tutoría docente de forma adecuada. Ese mismo procedimiento se aplicó en las cinco hipótesis específicas cuyos resultados determinaron una correlación de $\mathrm{Rho}=.492$, Rho $=.656$, Rho $=.464$, Rho $=.670$ y Rho $=581$. Conclusiones: La mayoria de los estudiantes consideran aceptable la regularidad con el cual se aplican las estrategias de enseñanza los cuales inciden en la tutoría docente de forma adecuada según la opinión de la mayoría de la muestra, sin embargo aún no existe una satisfacción total por parte de la muestra.

Palabras clave: Estrategias de enseñanza, tutoría docente, conocimientos previos, orientación, codificación, organización.

\begin{abstract}
Objective: To demonstrate the relationship between Teaching Strategies and Teaching Tutoring in students IX cycle of the Higher Public Technological Institute Huando - Huaral - Lima, 2018. Material and Methods: The quantitative approach of correlational design was raised, whose information was collected at through two questionnaires applied to the sample made up of 57 students. Likewise, its reliability was guaranteed by means of the Cronbach's Alpha test, in addition to applying the SPSS version 21 computational package, with the use of Spearman's Rho formula with a margin of error of $5 \%$. Results: The general hypothesis represented by the study variables found a good correlation of Rho $=.734$, since the majority considered that the teaching strategies are almost always applied, which affects the teaching tutoring adequately. The same procedure was applied in the five specific hypotheses whose results determined a correlation of Rho $=.492$, Rho $=.656$, Rho $=.464$, Rho $=.670$ and Rho $=$ 581. Conclusions: Most students consider acceptable regularity with which the teaching strategies are applied which affect the teaching tutoring in an appropriate way according to the opinion of the majority of the sample, however there is still no total satisfaction on the part of the sample
\end{abstract}

Keywords: Teaching strategies, teacher tutoring, prior knowledge, guidance, coding, organization.

\section{INTRODUCCIÓN}

El presente estudio se realizó alentado a mejorar el nivel de aplicabilidad de las estrategias de enseñanza en las universidades, estas metodologías de ayuda para ampliar y fortalecer los conocimientos de los estudiantes son fundamentales hoy en día puesto que se tiene que proveer de las herramientas necesarias para afrontar su entorno laboral que por si es muy competitiva, es por eso que el estudiante también se vuelve más exigente con lo espera de la universidad y de los docentes.

EI INEI (2014) realizó un análisis de sobre el contexto educativo universitario en donde presentaron algunas percepciones de los egresados universitarios sobre el desempeño académico de los docentes en las universidades públicas, en los cuales se destaca que la preparación de la clase de los docentes es buena para un $46.4 \%$ seguido de $6.3 \%$ que lo identifica como excelente, otro aspecto que se toma en consideración es el grado de actualización de los conocimientos en donde el $46.4 \%$ lo califica de bueno seguido de un $8.6 \%$ como excelente.

Con ello podemos observar que los estudiantes en su mayoría se sienten conforme por como organizan la clase y la preparación que tienen para ejercer su función, sin embargo, esta satisfacción aun no supera las expectativas de los futuros profesionales, ya que en ninguno de estas perspectivas el nivel excelente no alcanza ni el $10 \%$, sin considerar los otros porcentajes que manifiestan insatisfacción, es decir aún existen factores que analizar, entre estos las estrategias de enseñanza necesarios para organizar las clase.

No todos los estudiantes se adaptan a una determinada metodología académica, sin embargo, debemos ser conscientes que no debemos descuidar la función tutora que nos permite estar alerta de estas dificultades y plantear alternativas que garanticen un equilibrio en la efectividad positiva de las estrategias de enseñanza en el aula. Desde esta perspectiva, el MINEDU (2007) lo describe como un servicio de acompañamiento cognitivo y pedagógico para la adecuada formación docente. Incluso, en la Ley Universitaria $N^{\circ} 30220$ enfatiza que una de las funciones del docente es la de brindar tutoría a los estudiantes para orientarlos en su desarrollo profesional y/o académico.

En otras palabras, el docente debe estar comprometido con el desarrollo profesional de los estudiantes, seleccionando con la estrategia de enseñanza que más adapte en el aula asignada, ya que su aplicabilidad permitirá que su competencia tutora permita orientar adecuadamente en base a los objetivos académicos, pero siempre se debe estar alerta ante las incidencias que pueda acontecer, lo cual lo hemos podido apreciar en la población en estudio.

\footnotetext{
1 Universidad Mayor de San Marcos.

Recibido: 27/11/2018 Aprobado: 29/01/2019
} 


\section{MATERIAL Y MÉTODOS}

Corresponde a la investigación del enfoque cuantitativo, nivel explicativo, puesto que se trata de verificar y evaluar la relación entre las variables existentes: Es tipo no experimental y diseño correlacional.

La población está conformada por 57 estudiantes del $\mathrm{V}$ ciclo del Instituto Superior Tecnológico Público Huando - Huaral Lima, 2018.

Se aplicó la técnica de la encuesta tipo cuestionario para la variable $\mathrm{X}$ : Estrategias de Enseñanza, igual para la variable Y: Tutoría Docente.

Para el tratamiento estadístico, se empleó el paquete computacional SPSS versión 21, para establecer la contrastación de las hipótesis.

\section{RESULTADOS}

Prueba de hipótesis general

Ha. Existe una relación significativa entre las Estrategias de Enseñanza y la Tutoría Docente en estudiantes $\mathrm{V}$ ciclo del Instituto Superior Tecnológico Público Huando - Huaral Lima, 2018.

Ho. No existe una relación significativa entre las Estrategias de Enseñanza y la Tutoría Docente en estudiantes $V$ ciclo del Instituto Superior Tecnológico Público Huando - Huaral Lima, 2018.

Tabla 1

Correlación de la hipotesis general

\begin{tabular}{lccc}
\hline \multicolumn{2}{l}{ Correlaciones } & & Tutoría doc. \\
\hline \multirow{2}{*}{$\begin{array}{l}\text { Rho de } \\
\text { Spearman }\end{array}$} & Estrategias & de enseñante & de correlación \\
& & Sig. (bilateral) & 0,734 \\
& & $\mathrm{~N}$ & 57,000 \\
\hline
\end{tabular}

Interpretación. Al aplicar la fórmula de Rho de Spearman, margen de error al $5 \%$, se halló un coeficiente de correlación buena de $\mathrm{Rho}=0,734$ y el $p=0,000$. Por lo cual se rechaza la hipótesis nula y se acepta la hipótesis alterna, cuyos resultados fueron determinados por la respuesta de la muestra, en donde la mayoría considera que las Estrategias de Enseñanza, identificado mayoritariamente como casi siempre aplicadas en sus actividades académicas, interfiere de forma adecuada en la tutoría docente.

\section{Contrastación de la primera hipótesis específica}

H1. Existe una relación significativa entre las Estrategias de activación de los conocimientos previos y la Tutoría Docente en estudiantes IX ciclo del Instituto Superior Tecnológico Público Huando - Huaral - Lima, 2018.

Ho. No existe una relación significativa entre las Estrategias de activación de los conocimientos previos y la Tutoría Docente en estudiantes IX ciclo del Instituto Superior Tecnológico Público Huando - Huaral - Lima, 2018.
Tabla 2

Correlación de la primera hipótesis específica

\begin{tabular}{lccc}
\hline Correlaciones & & $\begin{array}{c}\text { Tutoría } \\
\text { docente }\end{array}$ \\
\hline Rho de & $\begin{array}{c}\text { Activación de los } \\
\text { conocimientos } \\
\text { previos }\end{array}$ & $\begin{array}{c}\text { Coeficiente de } \\
\text { correlación }\end{array}$ & 0,492 \\
Spearman & $\begin{array}{c}\text { Sig. (bilateral) } \\
\mathrm{N}\end{array}$ & $\begin{array}{c}0,000 \\
57\end{array}$ \\
\hline
\end{tabular}

Interpretación: Al aplicar la fórmula de Rho de Spearman, margen de error al $5 \%$, se halló un coeficiente de correlación moderada de $R$ ho $=0,492$ y el $p=0,000$. Por lo cual se rechaza la hipótesis nula y se acepta la hipótesis alterna, cuyos resultados fueron determinados por la respuesta de la muestra, en donde la mayoría considera que las Estrategias de Activación de los conocimientos previos, identificado mayoritariamente como casi siempre aplicadas en sus actividades académicas, interfiere adecuadamente en la tutoría docente.

\section{Contrastación de la segunda hipótesis específica}

H2. Existe una relación significativa entre las Estrategias de Orientación y guía relevante a los contenidos y la tutoría docente en estudiantes IX ciclo del Instituto Superior Tecnológico Público Huando - Huaral - Lima, 2018.

Ho. Existe una relación significativa entre las Estrategias de Orientación y guía relevante a los contenidos y la tutoría docente en estudiantes IX ciclo del Instituto Superior Tecnológico Público Huando - Huaral - Lima, 2018.

\section{Tabla 3}

Correlación de la segunda hipótesis especifica

\begin{tabular}{lccc}
\hline \multicolumn{3}{l}{ Correlaciones } & Orientación \\
\hline Rho de & $\begin{array}{c}\text { Coeficiente de } \\
\text { correlación } \\
\text { Spearman } \\
\text { relevante a los } \\
\text { contenidos }\end{array}$ & $\begin{array}{c}\text { Sig. (bilateral) } \\
0,656\end{array}$ \\
\hline
\end{tabular}

Interpretación: Al aplicar la fórmula de Rho de Spearman, margen de error al $5 \%$, se halló un coeficiente de correlación buena de $R$ ho $=0,656$ y el $p=0,000$. Por lo cual se rechaza la hipótesis nula y se acepta la hipótesis alterna, cuyos resultados fueron determinados por la respuesta de la muestra, en donde la mayoría considera que las Estrategias de Orientación y guía relevante a los contenidos, identificado mayoritariamente como casi siempre aplicadas en sus actividades académicas, interfiere adecuadamente en la tutoría docente.

\section{Contrastación de la tercera hipótesis específica}

H3. Existe una relación significativa entre las Estrategias de Mejoramiento en la codificación de la información y la Tutoría Docente en estudiantes IX ciclo del Instituto Superior Tecnológico Público Huando - Huaral - Lima, 2018

Ho. No existe una relación significativa entre las Estrategias de Mejoramiento en la codificación de la información y la Tutoría Docente en estudiantes IX ciclo del Instituto Superior Tecnológico Público Huando - Huaral - Lima, 2018 
Tabla 4

Correlación de la tercera hipótesis especifica

\begin{tabular}{lccc}
\hline Correlaciones & & $\begin{array}{c}\text { Tutoría } \\
\text { docente }\end{array}$ \\
\hline Rho de & Mejoramiento en & $\begin{array}{c}\text { Coeficiente de } \\
\text { correlación }\end{array}$ & 0,464 \\
Spearman & $\begin{array}{c}\text { la codificación de } \\
\text { la información }\end{array}$ & $\begin{array}{c}\text { Sig. (bilateral) } \\
\text { la }\end{array}$ & 0,000 \\
& $\mathrm{~N}$ & 57 \\
\hline
\end{tabular}

Interpretación: Al aplicar la fórmula de Rho de Spearman, margen de error al $5 \%$, se halló un coeficiente de correlación moderada de $R$ ho $=0,464$ y el $p=0,000$. Por lo cual se rechaza la hipótesis nula y se acepta la hipótesis alterna, cuyos resultados fueron determinados por la respuesta de la muestra, en donde la mayoría considera que las Estrategias de Mejoramiento en la codificación de la información, identificado mayoritariamente como casi siempre aplicadas en sus actividades académicas, interfiere adecuadamente en la tutoría docente.

\section{Contrastación de la cuarta hipótesis específica}

H4. Existe una relación significativa entre las Estrategias para organizar la información nueva por aprender y la Tutoría Docente en estudiantes IX ciclo del Instituto Superior Tecnológico Público Huando - Huaral - Lima, 2018.

Ho. No existe una relación significativa entre las Estrategias para organizar la información nueva por aprender y la Tutoría Docente en estudiantes IX ciclo del Instituto Superior Tecnológico Público Huando - Huaral - Lima, 2018.

Tabla 5

Correlación de la cuarta hipótesis especifica

\begin{tabular}{|c|c|c|c|}
\hline \multicolumn{3}{|c|}{ Correlaciones } & $\begin{array}{l}\text { Tutoría } \\
\text { docente }\end{array}$ \\
\hline \multirow{3}{*}{$\begin{array}{l}\text { Rho de } \\
\text { Spearman }\end{array}$} & \multirow{3}{*}{$\begin{array}{c}\text { Estrategias } \\
\text { para organizar } \\
\text { la información } \\
\text { nueva por } \\
\text { aprender }\end{array}$} & $\begin{array}{c}\text { Coeficiente } \\
\text { de } \\
\text { correlación }\end{array}$ & 0,670 \\
\hline & & Sig. (bilateral) & 0,000 \\
\hline & & $\mathrm{N}$ & 57 \\
\hline
\end{tabular}

Interpretación: Al aplicar la fórmula de Rho de Spearman, margen de error al $5 \%$, se halló un coeficiente de correlación buena de $\mathrm{Rho}=0,670$ y el $p=0,000$. Por lo cual se rechaza la hipótesis nula y se acepta la hipótesis alterna, cuyos resultados fueron determinados por la respuesta de la muestra, en donde la mayoría considera que las Estrategias para organizar la información nueva por aprender, identificado mayoritariamente como casi siempre aplicadas en sus actividades académicas, interfiere adecuadamente en la tutoría docente.

\section{Contrastación de la quinta hipótesis específica}

H5. Existe una relación significativa entre las Estrategias que promueven enlace entre conocimientos previos y nueva información, y la Tutoría Docente en estudiantes IX ciclo del Instituto Superior Tecnológico Público Huando - Huaral Lima, 2018.

Ho. No existe una relación significativa entre las Estrategias que promueven enlace entre conocimientos previos y nueva información, y la Tutoría Docente en estudiantes IX ciclo del Instituto Superior Tecnológico Público Huando - Huaral Lima, 2018.
Tabla 6

Correlación de la quinta hipótesis especifica

\begin{tabular}{|c|c|c|c|}
\hline \multicolumn{3}{|c|}{ Correlaciones } & $\begin{array}{r}\text { Tutoría } \\
\text { docente }\end{array}$ \\
\hline \multirow{3}{*}{$\begin{array}{l}\text { Rho de } \\
\text { Spearman }\end{array}$} & \multirow{3}{*}{$\begin{array}{c}\text { Promueven } \\
\text { enlace entre } \\
\text { conocimientos } \\
\text { previos y nueva } \\
\text { información }\end{array}$} & $\begin{array}{l}\text { Coeficiente de } \\
\text { correlación }\end{array}$ & 0,581 \\
\hline & & Sig. (bilateral) & 0,000 \\
\hline & & $\mathrm{N}$ & 57 \\
\hline
\end{tabular}

Interpretación: Al aplicar la fórmula de Rho de Spearman, margen de error al 5\%, se halló un coeficiente de correlación moderada de $R h o=0,581$ y el $p=0,000$. Por lo cual se rechaza la hipótesis nula y se acepta la hipótesis alterna, cuyos resultados fueron determinados por la respuesta de la muestra, en donde la mayoría considera que las estrategias que promueven enlace entre conocimientos previos y nueva información, identificado mayoritariamente como casi siempre aplicadas en sus actividades académicas, interfiere adecuadamente en la tutoría docente.

\section{DISCUSIÓN}

La hipótesis general determinó estadísticamente un coeficiente de correlación buena de Rho $=0,734$, entre las variables Estrategias de Enseñanza y la Tutoría Docente en base a las perspectivas de los estudiantes de la población en estudio. Se encontró discrepancias en las respuestas de la muestra, representadas en la variable Estrategias de Enseñanza cuyas respuestas determinaron que el 50,9\% considera son aplicadas casi siempre, seguido de un $35,1 \%$ que lo identifica como a veces. Asimismo, para la variable Tutoría Docente cuyas respuestas también se encuentran divididas, puesto que el $50,9 \%$ lo considera adecuado, mientras que un $31,6 \%$ lo refiere como a veces adecuado. Con ello se concluye que las Estrategias de Enseñanza son aplicadas con una frecuencia aceptable para la mayoría de la población de estudio por lo cual la tutoría docente es más apreciada por ellos porque cumple adecuadamente con las necesidades académicas de los estudiantes, sin embargo, aún existe un importante porcentaje de la población que manifiesta una satisfacción media.

Con respecto a la primera hipótesis específica se estableció en las estadísticas un coeficiente de correlación moderada de Rho $=0,492$ entre las Estrategias de Activación de los conocimientos previos y la variable Tutoría Docente en base a las perspectivas de los estudiantes de la población en estudio. Se pudo encontrar diferencias en la muestra, representadas en la dimensión Estrategias de Activación de los conocimientos previos cuyas respuestas determinaron que el $49,1 \%$ lo identifica como casi siempre, seguido de un $24,6 \%$ que lo refiere como casi siempre. Del mismo modo, para la variable Tutoría Docente cuyas respuestas también se encuentran divididas, puesto que el $50,9 \%$ lo considera adecuado, mientras que un $31,6 \%$ lo refiere como a veces adecuado. Con ello se confirma que las Estrategias de Activación de los conocimientos previos son aplicadas con una frecuencia aceptable para la mayoría de la población de estudio por lo cual la Tutoría Docente es más apreciada por ellos porque cumple adecuadamente con las necesidades académicas de los estudiantes, sin embargo, aún existe un importante porcentaje de la población que manifiesta una satisfacción media.

En la segunda hipótesis específica, las estadísticas encontraron un coeficiente de correlación buena de Rho = 
0,656, entre la dimensión Estrategias de Orientación y guía relevante a los contenidos y la Tutoría Docente en base a las perspectivas de los estudiantes de la población en estudio. Encontrándose diferencias en las respuestas de la muestra en cuanto a la dimensión Estrategias de Orientación y guía relevante a los contenidos cuyas respuestas determinaron que el $47,4 \%$ lo refiere como casi siempre, seguido de un $29,8 \%$ que lo identifica como a veces. Con el mismo procedimiento, para la variable Tutoría Docente cuyas respuestas también se encuentran divididas, puesto que el $50,9 \%$ lo considera adecuado, mientras que un $31,6 \%$ lo refiere como a veces adecuado. Por ello, se confirma que las Estrategias de Orientación y guía relevante a los contenidos son aplicadas con una frecuencia aceptable para la mayoría de la población de estudio por lo cual la Tutoría Docente es más apreciada por ellos porque cumple adecuadamente con las necesidades académicas de los estudiantes, sin embargo, aún existe un importante porcentaje de la población que manifiesta una satisfacción media.

En la tercera hipótesis específica, las estadísticas hallaron un coeficiente de correlación moderada de Rho $=0,464$, entre la dimensión Estrategias de Mejoramiento en la codificación de la información y la Tutoría Docente en base a las percepciones de los estudiantes. Encontrándose diferencias en las respuestas de la muestra en cuanto a la dimensión Estrategias de Mejoramiento en la codificación de la información en el cual precisaron que el $42,1 \%$ lo identifica como casi siempre, seguido de un $38.6 \%$ que lo refiere como a veces. Asimismo, para la variable para la variable Tutoría Docente cuyas respuestas también se encuentran divididas, puesto que el $50,9 \%$ lo considera adecuado, mientras que un $31,6 \%$ lo refiere como a veces adecuado. Evidenciándose que las Estrategias de Mejoramiento en la codificación de la información son aplicadas con una frecuencia aceptable para la mayoría de la población de estudio por lo cual la Tutoría Docente es más apreciada por ellos porque cumple adecuadamente con las necesidades académicas de los estudiantes, sin embargo, aún existe un importante porcentaje de la población que manifiesta una satisfacción media.

Con respecto a la cuarta hipótesis específica, las estadísticas hallaron un coeficiente de correlación buena de Rho = 0,670 , entre la dimensión Estrategias para organizar la información nueva por aprender y la Tutoría Docente en base a las percepciones de los estudiantes. Encontrándose discrepancias en las respuestas de la muestra en cuanto a la dimensión Estrategias para organizar la información nueva por aprender en el cual precisaron que el 40,4\% lo identifica como casi siempre, seguido de un $33,3 \%$ que lo refiere como a veces. En cuanto a la variable Tutoría Docente las respuestas también manifestaron discrepancias, puesto que el 50,9\% lo considera adecuado, mientras que un $31,6 \%$ lo refiere como a veces adecuado. Evidenciándose que las Estrategias para organizar la información nueva por aprender son aplicadas con una frecuencia aceptable para la mayoría de la población de estudio por lo cual la Tutoría Docente es más apreciada por ellos porque cumple adecuadamente con las necesidades académicas de los estudiantes, sin embargo, aún existe un importante porcentaje de la población que manifiesta una satisfacción media.

En la quinta hipótesis específica, las estadísticas hallaron un coeficiente de correlación moderada de Rho $=0,581$, entre la dimensión Estrategias que Promueven enlace entre conocimientos previos y nueva información, y la Tutoría Docente en base a las percepciones de los estudiantes. Encontrándose diferencias en las respuestas de la muestra en cuanto a la dimensión Estrategias que Promueven enlace entre conocimientos previos y nueva información en el cual precisaron que el $40,4 \%$ lo identifica como casi siempre, seguido de un $35,1 \%$ que lo refiere como a veces. Asimismo, en la variable Tutoría Docente las respuestas también manifestaron discrepancias, puesto que el 50,9\% lo considera adecuado, mientras que un $31,6 \%$ lo refiere como a veces adecuado. Concluyendo que las Estrategias que Promueven enlace entre conocimientos previos y nueva información son aplicadas con una frecuencia aceptable para la mayoría de la población de estudio por lo cual la Tutoría Docente es más apreciada por ellos porque cumple adecuadamente con las necesidades académicas de los estudiantes, sin embargo, aún existe un importante porcentaje de la población que manifiesta una satisfacción media.

Resultados similares, fueron los que encontró Martínez (2015) quien en su investigación logró determinar que las estrategias de enseñanza tienen una fuerte relación con los aprendizajes de los estudiantes, sin embargo, este dependerá de las características, diferencias y finalidad del grupo de estudio. En la presente investigación hemos notado que las estrategias de enseñanza no siempre son aplicadas en el aula ocasionando no solo la insatisfacción de un porcentaje del estudiante, sino se pierde la adaptabilidad de trabajo con una determinada metodología, incluso perjudicando a los que lentamente se van adaptando. En otras palabras, si las estrategias de enseñanza ayudan a una mayor efectividad de aprendizaje en los estudiantes, no todos se adaptan a esta por lo cual su frecuencia de aplicación debe ser constante y guiada para alcanzar los resultados esperados.

En cuanto a la tutoría docente, Comezaña (2013) establece en sus investigaciones que la tutoría docente cumple las expectativas de los estudiantes cuando existe buen trato y disposición para atender sus necesidades, asimismo este proviene de una adecuada aplicación de estrategias, resultados que también hemos comprobado fueron comprobados en la presente investigación, en donde a mayor aplicación de las estrategias de enseñanza existe mayor posibilidad de aplicar adecuadamente la función tutora, puesto que se está atento a su necesidades y dificultades durante su formación.

\section{CONCLUSIONES}

1. Los resultados de las variables Estrategias de Enseñanza y la Tutoría Docente, no alertan muchos inconvenientes, puesto que las Estrategias de Enseñanza son identificados como casi siempre, lo cual ha permitido que la Tutoría Docente sea desarrollada de forma adecuada, especialmente en el proceso de aprendizaje del alumno. Por ello, se concluye que a pesar de que la mayoría de estudiantes consideran aceptable la regularidad con la cual se aplican las estrategias de enseñanza, incluso incidiendo favorablemente en la tutoría docente, aún no existe una satisfacción total por parte de la muestra.

2. Entre la variable dimensión Estrategias de Activación de los conocimientos previos y la variable Tutoría Docente resultados relativamente favorables, ya que las Estrategias de activación de los conocimientos previos son aplicados casi siempre lo cual incide en la tutoría docente de forma adecuada, aunque con menor intensidad en la resolución de conflictos. Hallándose que a pesar de que la mayoría de estudiantes consideran aceptable la regularidad con la cual se aplican la actividad introductoria focal, la discusión guiada, la creación de lista de ideas y la explicación de los objetivos de las actividades, incluso incidiendo favorablemente en la tutoría docente, aún no existe una satisfacción total por 
parte de la muestra.

3. La correlación entre la dimensión Estrategias de Orientación y guía relevante a los contenidos y la variable Tutoría Docente, encontraron resultados relativamente esperados, puesto que las Estrategias de Orientación y guía relevante a los contenidos son aplicados casi siempre lo cual incide en la Tutoría Docente de forma adecuada, como en la búsqueda de fuentes documentales necesarios para las actividades de esta estrategia. Determinándose que a pesar de que la mayoría de estudiantes consideran aceptable la regularidad con la cual se aplican el énfasis y organización contenido relevante, los textos aclaratorios y la ayuda a la identificación de conceptos de interés, incluso incidiendo favorablemente en la tutoría docente, aún no existe una satisfacción total por parte de la muestra.

4. Con respecto a la dimensión Estrategias de Mejoramiento en la codificación de la información y la variable Tutoría Docente, los resultados no alertan muchos inconvenientes, ya que las Estrategias de Mejoramiento en la codificación de la información son aplicados casi siempre lo cual incide en la Tutoría Docente de forma adecuada, como en la guía para afrontar las dificultades del aprendizaje, necesarios para las actividades de esta estrategia. Determinándose que a pesar de que la mayoría de estudiantes consideran aceptable la regularidad con la cual se hacen uso de las ilustraciones, las gráficas y las preguntas intercaladas, incluso incidiendo favorablemente en la tutoría docente, aún no existe una satisfacción total por parte de la muestra.

5. En cuanto a la dimensión Estrategias para Organizar la información nueva por aprender y la variable Tutoría Docente, los resultados no alertan muchos inconvenientes, puesto que las Estrategias para Organizar la información nueva son aplicados casi siempre lo cual incide en la Tutoría Docente de forma adecuada, como en la metodología y trabajos de investigación, necesarios para las actividades desarrolladas esta estrategia. Determinándose que a pesar de que la mayoría de estudiantes consideran aceptable la regularidad con la cual se hacen uso de los resúmenes, los Organizadores gráficos y los mapas conceptuales, incluso incidiendo favorablemente en la tutoría docente, aún no existe una satisfacción total por parte de la muestra.

6. En el análisis de la dimensión Estrategias que promueven enlace entre conocimientos previos y nueva información, y la Tutoría Docente, no se manifiestan muchos inconvenientes, puesto que las Estrategias que promueven enlace entre conocimientos previos y nueva información son aplicados casi siempre lo cual incide en la Tutoría Docente de forma adecuada, como en la resolución de conflictos, necesarios para las actividades desarrolladas esta estrategia. Determinándose que a pesar de que la mayoría de estudiantes consideran aceptable la regularidad con la cual se hacen uso de los organizadores previos y la sugerencia de textos, incluso incidiendo favorablemente en la tutoría docente, aún no existe una satisfacción total por parte de la muestra.

\section{RECOMENDACIONES}

1. A las autoridades del Instituto Superior Tecnológico Público Huando se les sugiere realizar monitoreos periódicos a los docentes para verificar si las estrategias de enseñanza están siendo bien recibidas por los estudiantes, puesto de la aplicación de estas dependerá la función tutora del docente.

2. A los docentes se les sugiere recibir opiniones y absolver dudas con respecto al desarrollo de las estrategias de activación de conocimientos previos, ya que no todos los estudiantes se adaptan a determinada estrategia, de igual forma este contacto y preocupación para garantizar los aprendizajes de los estudiantes los incentivarán a una mejor función tutora.

3. Se recomienda a los estudiantes ser más proactivos en las clases, proponiendo y compartiendo sus perspectivas sobre la forma en la en las estrategias de orientación y guía relevante a los contenidos, ya que, si la metodología no cumple sus expectativas, el proceso de aprendizaje se dificultará por ausencia de la guía docente.

4. A las autoridades de la población de estudio mejorar e implementar las herramientas, equipos audiovisuales y áreas que sean necesarias para que los docentes puedan hacer uso de las ayudas visuales para el desarrollo didáctico de la clase, con las cuales se pueda mejorar la codificación de la información, de esta manera seguir reforzando la función tutora de los docentes.

5. A los estudiantes, cumplir con las actividades asignadas por el docente con respecto a las estrategias de organización de información nueva por aprender, y comunicar al docente si se presenta alguna dificultas, del mismo el docente en su función tutora debe trabajar y fortalecer su empatía.

6. Los docentes son conscientes de que los horarios académicos son muy cortos, por ende, las horas no son los suficientes para abarcar ampliamente todo lo que un determinado curso necesita. Es por eso que el profesional de la educación debe recomendar textos y lectura que fortalezcan los temas tratados en clase, no solo por el hecho que estos impliquen una actividad calificada, sino de lectura que los ayude en su profesión, el mismos mismo que le debe ir reforzando con la comunicación empática propios de la tutoría.

\section{AGRADECIMIENTOS:}

Agradezco a Dios por el don la vida, por guiarnos a lo largo de nuestra existencia, ser el apoyo y fortaleza en aquellos momentos de dificultad y de debilidad. Gracias a mis padres que Dios los tenga en su gloria: Casimiro y Norma; a mi esposo Marco Antonio y mi hija Alexandra, por ser los principales promotores de mis sueños, por confiar y creer en mis expectativas, y mi superación profesional.

Agradezco al Dr. Alfonso Vigo Quiñones Director del prestigioso Instituto de Educación Superior Tecnológico "Huando" y a sus estudiantes por haber permitido desarrollar mi proyecto de investigación, a mi asesor, Dr. Abelardo Campana Concha quien me ha guiado con paciencia, y sabiduría el presente trabajo que hoy se hace realidad, y finalmente agradezco al Universidad Nacional José Faustino Sánchez Carrión, a su prestigiosa revista BIG BANG, por la publicación del presente artículo. 


\section{REFERENCIAS BIBLIOGRÁFICAS}

Comezaña, K. (2013). La gestión tutorial, según el reporte del docente y su relación con el nivel de satisfacción de los estudiantes de secundaria (Tesis Posgrado) USMP, Lima, Perú.

INEI (2015). Encuesta Nacional a Egresados Universitarios y Universidades, 2014. Perú: Instituto Nacional de Estadística e Informática.

Martínez, P. (2015). Estrategias de enseñanza y nivel de aprendizaje en el área de comunicación en los estudiantes del cuarto de secundaria en la institución educativa "Mixto San Luis", provincia de Cañete, 2013 (Tesis Posgrado) Universidad Nacional de Educación, Lima, Perú.

MINEDU (2007). Manual tutoría y orientación educativa. Perú:

Ley $N^{\circ}$ 30220. Diario Oficial El Peruano, Lima, Perú, 09 de julio de 2017. 